\title{
SPHERICAL HALL ALGEBRAS OF CURVES AND HARDER-NARASIMHAN STRATAS
}

\author{
Olivier Schiffmann
}

\begin{abstract}
We show that the characteristic function $\mathbf{1}_{S_{\alpha}}$ of any HarderNarasimhan strata $S_{\alpha} \subset C o_{X}^{\alpha}$ belongs to the spherical Hall algebra $\mathbf{H}_{X}^{s p h}$ of a smooth projective curve $X$ (defined over a finite field $\mathbb{F}_{q}$ ). We prove a similar result in the geometric setting: the intersection cohomology complex $I C\left(\underline{S}_{\alpha}\right)$ of any Harder-Narasimhan strata $\underline{S}_{\alpha} \subset C \operatorname{Coh} \frac{\alpha}{\mathrm{X}}$ belongs to the category $\mathcal{Q}_{X}$ of spherical Eisenstein sheaves of $X$. We show by a simple example how a complete description of all spherical Eisenstein sheaves would necessarily involve the Brill-Noether stratas of $\underline{C o h} \frac{\alpha}{X}$.
\end{abstract}

\section{Introduction}

Let $X$ be a smooth projective curve defined over a finite field $\mathbb{F}_{q}$. To such a curve is associated, through a general formalism developed by Ringel, a Hopf algebra $\mathbf{H}_{X}$ (called the Hall algebra of $X$ ). As a vector space, $\mathbf{H}_{X}$ consists of all finitely supported functions on the set of (isomorphism classes of) coherent sheaves over $X$, and the (co)product encodes the structure of the extensions between coherent sheaves over $X$ (see e.g. [15]).

Hall algebras were first considered by Ringel in the context of representations of quivers. He showed that a certain natural subalgebra $\mathbf{C}_{\vec{Q}}$ of the Hall algebra $\mathbf{H}_{\vec{Q}}$ of a quiver $\vec{Q}$ is isomorphic to the quantized enveloping algebra $\mathbf{U}_{q}^{+}(\mathfrak{g})$ of a Kac-Moody algebra $\mathfrak{g}$ attached to $\vec{Q}$ ([13]). This discovery paved the way for a completely new approach to the theory of quantum groups based on the representation theory of quivers (see e.g. [7], [11], [21],...). One of the most important development is the work of Lusztig who considered a geometric version of the Hall algebra of a quiver, in which functions are replaced by constructible sheaves (on moduli stacks); this gave rise to the theory canonical bases of quantum groups, whose impact in algebraic and geometric representation theory is well-known (see [10]).

Received February 8, 2010.

2010 Mathematics Subject Classification. 17B37, $22 \mathrm{E} 57$.

Key words and phrases. Hall algebras, Harder-Narasimhan stratas, Eisenstein sheaves. 
In the context of smooth projective curves, Hall algebras first appeared in pioneering work of Kapranov in relation to the theory of automorphic forms over function fields (see [6]). He observed some striking similarities between the Hall algebras of curves and quantum affine algebras $\mathbf{U}_{q}^{+}(\widehat{\mathfrak{g}})$ (more precisely between the functional equations for Eisenstein series over function fields and the so-called Drinfeld relations in quantum affine algebras). This analogy becomes very precise for $X=\mathbb{P}^{1}$ (see [2]). Motivated by the theory of quantum loop algebras, we introduced in [14] a natural subalgebra $\mathbf{H}_{X}^{s p h}$ of $\mathbf{H}_{X}$ which we call the spherical Hall algebra of $X$; in the language of automorphic forms, $\mathbf{H}_{X}^{s p h}$ is generated by the Fourier coefficients of all Eisenstein series induced from the trivial character of a maximal torus. Inspired by work of Laumon, we also singled out a category $\mathcal{Q}_{X}$ of simple perverse sheaves over the moduli stacks $\operatorname{Coh}_{X}^{r, d}$ of coherent sheaves over $\bar{X}=X \otimes \overline{\mathbb{F}_{q}}$. These simple perverse sheaves (which we call spherical Eisenstein sheaves) are expected to provide (by means of the Faisceaux-Fonctions correspondence) a canonical basis for the spherical Hall algebras $\mathbf{H}_{X}^{s p h}$. From [19] it is also natural to expect $\mathcal{Q}_{X}$ to play a role in the geometric Langlands program for local systems in the formal neighborhood of the trivial local system.

The spherical Hall algebras $\mathbf{H}_{X}$ and the spherical Eisenstein sheaves $\mathcal{Q}_{X}$ of several low-genus (possibly orbifold) curves were computed in a series of papers (see [14], [4], [16], [17]) where they were shown to yield interesting quantum loop algebras (such as quantum toroidal algebras or spherical Cherednik algebras of type $A$ ) equipped with some canonical bases. Much less is known for higher genus curves; a combinatorial realization of $\mathbf{H}_{X}^{s p h}$ as a shuffle algebra is given in [19] for an arbitrary curve, but it is rather hard to analyze directly algebraically.

In this note, as a first step towards understanding these higher genus spherical Hall algebras, we exhibit an explicit class of elements in $\mathbf{H}_{X}^{s p h}$ when $X$ is of genus $g>1$. Namely we prove (see Theorem 3.1) that the characteristic functions of all the Harder-Narasimhan stratas $S_{\underline{\alpha}}$ belong to $\mathbf{H}_{X}^{s p h}$ (see Section 2 for notations). We also give a geometric version of the same result: the intersection cohomology complex $I C\left(\underline{S}_{\alpha}\right)$ of any Harder-Narasimhan strata is a spherical Eisenstein sheaf (Theorem 5.1 ). As we show by an example, these classes of functions (resp. simple perverse sheaves) come very far from exhausting the whole of $\mathbf{H}_{X}^{\text {sph }}$ (resp. $\mathcal{Q}_{X}$ ): a full description would (at least) involve the various Brill-Noether loci in $\underline{C o h}_{X}^{r, d}$ (see Remark 5.3).

\section{Spherical Hall algebras of curves}

1.1. Let $X$ be a connected smooth projective curve of genus $g$ defined over the finite field $\mathbb{F}_{q}$. We will assume here that $g>1$, although most of the results proved in this note hold for rational and elliptic curves as well (see [16], $[17])$. Let $\operatorname{Coh}(X)$ stand for the category of coherent sheaves over $X$. Let us denote by $\langle$,$\rangle the Euler form on the Grothendieck group K_{0}(X)$, and let $K_{0}^{\prime}(X)=K_{0}(X) / \operatorname{rad}\langle$,$\rangle be the numerical Grothendieck group of X$. We have 
$K_{0}^{\prime}(X)=\mathbb{Z} \mathrm{rk} \oplus \mathbb{Z}$ deg, where rk and deg are the rank and degree functions respectively. The Euler form on $K_{0}(X)$ is given by

$$
\langle\mathcal{F}, \mathcal{G}\rangle=(1-g) \operatorname{rk}(\mathcal{F}) \operatorname{rk}(\mathcal{G})+\left|\begin{array}{cc}
\operatorname{rk}(\mathcal{F}) & \operatorname{rk}(\mathcal{G}) \\
\operatorname{deg}(\mathcal{F}) & \operatorname{deg}(\mathcal{G})
\end{array}\right| .
$$

1.2. Let us briefly recall the definition of the Hall algebra $\mathbf{H}_{X}$ of $X$. We refer the reader to [15, Lecture 4] for more details. Let $\mathcal{I}_{X}$ stand for the set of all coherent sheaves over $X$. Put

$$
\mathbf{H}_{X}=\left\{f: \mathcal{I}_{X} \rightarrow \mathbb{C} \mid \# \operatorname{supp} f<\infty\right\}=\bigoplus_{\mathcal{F} \in \mathcal{I}} \mathbb{C} 1_{\mathcal{F}},
$$

where $1_{\mathcal{F}}$ is the characteristic function of the point $\mathcal{F} \in \mathcal{I}$. Let us fix a square root $v$ of $q^{-1}$. The multiplication in $\mathbf{H}_{X}$ is defined by the following formula

$$
(f \cdot g)(\mathcal{R})=\sum_{\mathcal{N} \subseteq \mathcal{R}} v^{-\langle\mathcal{R} / \mathcal{N}, \mathcal{N}\rangle} f(\mathcal{R} / \mathcal{N}) g(\mathcal{N})
$$

and the comultiplication is

$$
\Delta(f)(\mathcal{M}, \mathcal{N})=\frac{v^{\langle\mathcal{M}, \mathcal{N}\rangle}}{\left|\operatorname{Ext}^{1}(\mathcal{M}, \mathcal{N})\right|} \sum_{\xi \in \operatorname{Ext}^{1}(\mathcal{M}, \mathcal{N})} f\left(\mathcal{X}_{\xi}\right),
$$

where $\mathcal{X}_{\xi}$ is the extension of $\mathcal{N}$ by $\mathcal{M}$ corresponding to $\xi$. Notice that the coproduct $\Delta$ takes values in a completion $\mathbf{H}_{X} \widehat{\otimes} \mathbf{H}_{X}$ of the tensor space $\mathbf{H}_{X} \otimes$ $\mathbf{H}_{X}$ only (see e.g. [4, Section 2]). The triple $\left(\mathbf{H}_{X}, \cdot, \Delta\right)$ is not a (topological) bialgebra, but it becomes one if we suitably twist the coproduct. For this we introduce an extra subalgebra $\mathcal{K}=\mathbb{C}\left[\boldsymbol{\kappa}_{r, d}\right],(r, d) \in \mathbb{Z}^{2}$, and we define an extended Hall algebra $\widetilde{\mathbf{H}}_{X}=\mathbf{H}_{X} \otimes \mathcal{K}$ with relations

$$
\boldsymbol{\kappa}_{r, d} \boldsymbol{\kappa}_{s, l}=\boldsymbol{\kappa}_{r+s, d+l}, \quad \boldsymbol{\kappa}_{0,0}=1, \quad \boldsymbol{\kappa}_{r, d} 1_{\mathcal{M}} \boldsymbol{\kappa}_{r, d}^{-1}=v^{-2 r(1-g) \operatorname{rk}(\mathcal{M})} 1_{\mathcal{M}} .
$$

The new coproduct is given by the formulas

$$
\begin{gathered}
\widetilde{\Delta}\left(\boldsymbol{\kappa}_{r, d}\right)=\boldsymbol{\kappa}_{r, d} \otimes \boldsymbol{\kappa}_{r, d}, \\
\widetilde{\Delta}(f)=\sum_{\mathcal{M}, \mathcal{N}} \Delta(f)(\mathcal{M}, \mathcal{N}) 1_{\mathcal{M}} \boldsymbol{\kappa}_{r_{\mathcal{N}}, d_{\mathcal{N}}} \otimes 1_{\mathcal{N}} .
\end{gathered}
$$

Then $\left(\widetilde{\mathbf{H}}_{X}, \cdot, \widetilde{\Delta}\right)$ is a topological bialgebra.

The Hall algebras $\mathbf{H}_{X}$ and $\widetilde{\mathbf{H}}_{X}$ are $\mathbb{Z}^{2}$-graded (by the class in the numerical Grothendieck group). We will sometimes write $\Delta_{\alpha, \beta}$ or $\widetilde{\Delta}_{\alpha, \beta}$ in order to specify the graded components of the coproduct. 
1.3. We will especially be interested in the spherical subalgebra $\mathbf{H}_{X}^{s p h}$ of $\mathbf{H}_{X}$, which is defined as follows. For any $d \in \mathbb{Z}$ let $\operatorname{Pic}^{d}(X)$ stand for the (finite) set of line bundles over $X$ of degree $d$, and let us set

$$
\mathbf{1}_{1, d}^{\mathrm{ss}}=\sum_{\mathcal{L} \in \operatorname{Pic}^{d}(X)} 1_{\mathcal{L}}
$$

Next, let $d \geq 1$ and let $\operatorname{Tor}^{d}(X)$ stand for the (finite) set of all torsion sheaves over $X$ of degree $d$. We now set

$$
\mathbf{1}_{0, d}=\sum_{\mathcal{T} \in \operatorname{Tor}^{d}(X)} 1_{\mathcal{T}}
$$

The spherical Hall algebra $\mathbf{H}_{X}^{s p h}$ is generated by the elements $\left\{\mathbf{1}_{1, d}^{\mathbf{s s}} \mid d \in\right.$ $\mathbb{Z}\} \cup\left\{\mathbf{1}_{0, d} \mid d \geq 1\right\}$. In an effort to unburden the notation, and because this should not cause any confusion here, we will simply write $\mathbf{U}_{X}$ for $\mathbf{H}_{X}^{s p h}$. The spherical Hall algebra contains two natural subalgebras, namely $\mathbf{U}_{X}^{>}$which is generated by $\left\{\mathbf{1}_{1, d}^{\text {ss }} \mid d \in \mathbb{Z}\right\}$, and $\mathbf{U}_{X}^{0}$ which is generated by $\left\{\mathbf{1}_{0, d} \mid d \geq 1\right\}$. Moreover, the multiplication map gives an isomorphism $\mathbf{U}_{X}^{>} \otimes \mathbf{U}_{X}^{0} \rightarrow \mathbf{U}_{X}$ (see e.g. $[18$, Section 6]).

\section{Harder-Narasimhan stratifications}

2.1. Let us now briefly recall the various notions related to semistability of coherent sheaves over curves. We refer to [5], [20] for more details. We fix a smooth projective curve $X$ of genus $g$ as in Section 1.1. The slope of a coherent sheaf $\mathcal{F}$ over $X$ is defined to be

$$
\mu(\mathcal{F})=\frac{\operatorname{deg}(\mathcal{F})}{\operatorname{rank}(\mathcal{F})} \in \mathbb{Q} \cup\{\infty\} .
$$

The sheaf $\mathcal{F}$ is said to be semistable of slope $\nu$ if $\mu(\mathcal{F})=\nu$ and if $\mu(\mathcal{G}) \leq \nu$ for any subsheaf $\mathcal{G}$ of $\mathcal{F}$. If the above condition holds with $<$ instead of $\leq$, then we say that $\mathcal{F}$ is stable. We denote by $\mathbf{C}_{\nu}$ the full subcategory of $\operatorname{Coh}(X)$ whose objects are semistable sheaves of slope $\nu$. The categories $\mathbf{C}_{\nu}$ are abelian and artinian. The simple objects of $\mathbf{C}_{\nu}$ are precisely given by the stable sheaves of slope $\nu$.

The fundamental properties of the categories $\mathbf{C}_{\nu}$ are listed below.

Proposition 2.1. The following hold:

i) $\operatorname{Hom}\left(\boldsymbol{C}_{\nu}, \boldsymbol{C}_{\eta}\right)=0$ if $\nu>\eta$,

ii) $\operatorname{Ext}\left(\boldsymbol{C}_{\nu}, \boldsymbol{C}_{\eta}\right)=0$ if $\eta>\nu+2(g-1)$,

iii) any coherent sheaf $\mathcal{F}$ possesses a unique filtration

$$
0 \subsetneq \mathcal{F}_{l} \subsetneq \cdots \subsetneq \mathcal{F}_{1}=\mathcal{F}
$$

satisfying the following conditions: $\mathcal{F}_{i} / \mathcal{F}_{i+1}$ is semistable for all $i$ and

$$
\mu\left(\mathcal{F}_{1} / \mathcal{F}_{2}\right)<\cdots<\mu\left(\mathcal{F}_{l-1} / \mathcal{F}_{l}\right)<\mu\left(\mathcal{F}_{l}\right) .
$$


The filtration (2.1) is called the Harder-Narasimhan (or HN) filtration of $\mathcal{F})$. We also define the $H N$-type of $\mathcal{F}$ to be $H N(\mathcal{F})=\left(\alpha_{1}, \ldots, \alpha_{l}\right)$ with $\alpha_{i}=$ $\overline{\mathcal{F}_{i}}-\overline{\mathcal{F}_{i+1}}$. Here $\overline{\mathcal{G}}=(\operatorname{rank}(\mathcal{G}), \operatorname{deg}(\mathcal{G})) \in \mathbb{Z}^{2}$ is the class of a sheaf $\mathcal{G}$ in the (numerical) Grothendieck group of $\operatorname{Coh}(X)$-see Section 1.1. Note that the weight $\alpha:=\alpha_{1}+\cdots+\alpha_{l}$ of the HN type of $\mathcal{F}$ is equal to $\overline{\mathcal{F}}$.

It is convenient to view an HN type $\left(\alpha_{1}, \ldots, \alpha_{l}\right)$ as a polygon as follows:

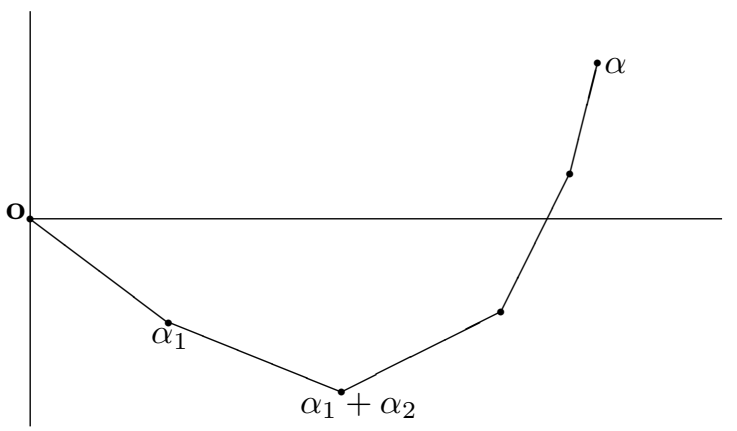

Figure 1. A Harder-Narasimhan polygon of weight $\alpha$.

This polygon, called the $H N$ polygon of $\mathcal{F}$, is convex by construction. The following useful result is a consequence of Proposition 2.1 (see e.g. [20, Theorem 2]).

Proposition 2.2. Let $\mathcal{F}$ be a coherent sheaf over $X$ of class $\alpha \in \mathbb{Z}^{2}$. Let $0 \subsetneq \mathcal{F}_{l} \subsetneq \cdots \subsetneq \mathcal{F}_{1}=\mathcal{F}$ be the $H N$ filtration of $\mathcal{F}$. Let $\mathcal{G}$ be a subsheaf of $\mathcal{F}$ of class $\gamma$. Then

i) the point $\beta:=\alpha-\gamma$ lies above the $H N$ polygon of $\mathcal{F}$,

ii) if moreover $\beta$ is a vertex of the $H N$ polygon of $\mathcal{F}$, that is if $\gamma=\alpha_{i}+$ $\cdots+\alpha_{l}$ for some $1 \leq i \leq l$, then $\mathcal{G}=\mathcal{F}_{i}$.

2.2. We may stratify the set $\mathcal{I}_{X}$ of all isomorphism classes of coherent sheaves over $X$ by the HN-type and write $\mathcal{I}_{X}=\bigsqcup_{\alpha} S_{\alpha}$ where $\underline{\alpha}$ runs through the set of all possible HN types, i.e., tuples $\underline{\alpha}=\left(\alpha_{1}, \ldots, \alpha_{l}\right)$ with $\alpha_{i} \in\left(\mathbb{Z}^{2}\right)^{+}$and $\mu\left(\alpha_{1}\right)<\cdots<\mu\left(\alpha_{l}\right)$. Here $\left(\mathbb{Z}^{2}\right)^{+}=\left\{(r, d) \in \mathbb{Z}^{2} \mid r \geq 1\right.$ or $\left.r=0, d>0\right\}$. For instance, if $\underline{\alpha}=(\alpha)$, then $S_{\alpha}$ is the set of isomorphism classes of semistable sheaves of class $\alpha$. Let us denote by $\mathbf{1}_{S_{\alpha}} \in \mathbf{H}_{X}$ the characteristic function of the set of sheaves of a fixed HN type $\underline{\alpha}$. Since $X$ is defined over a finite field, $S_{\underline{\alpha}}$ is finite for any $\underline{\alpha}$ hence $\mathbf{1}_{S_{\underline{\alpha}}}$ is a well-defined element of $\mathbf{H}_{X}$. For $\alpha \in\left(\mathbb{Z}^{2}\right)^{+}$ we will simply denote by $\mathbf{1}_{\alpha}^{\text {ss }}$ the characteristic function of $S_{\alpha}$.

From the uniqueness of the HN filtration of a given coherent sheaf we easily deduce 
Proposition 2.3. For any $H N$ type $\underline{\alpha}=\left(\alpha_{1}, \ldots, \alpha_{l}\right)$ we have

$$
\mathbf{1}_{S_{\underline{\alpha}}}=v^{\sum_{i<j}\left\langle\alpha_{i}, \alpha_{j}\right\rangle} \mathbf{1}_{\alpha_{1}}^{s s} \cdots \mathbf{1}_{\alpha_{l}}^{s s} .
$$

We use the stratification by HN type to define a completion of the Hall algebra $\mathbf{H}_{X}$ as follows. For $n \in \mathbb{Z}$ let us write $\underline{\alpha} \geq n$ if $\underline{\alpha}=\left(\alpha_{1}, \ldots, \alpha_{l}\right)$ with $\mu\left(\alpha_{1}\right) \geq n$. Let $\mathbf{C}_{\geq n}$ be the full subcategory of $\operatorname{Coh}(X)$ generated by $\mathbf{C}_{\nu}$ for all $\nu \geq n$. By definition, the HN type $\underline{\alpha}$ of a sheaf $\mathcal{F}$ satisfies $\underline{\alpha} \geq n$ if and only if $\mathcal{F} \in \mathbf{C}_{\geq n}$.

The set of HN types of a fixed weight $\alpha$ satisfying $\underline{\alpha} \geq n$ is finite for any $n$. Let $\mathbf{H}_{X}^{<n}[\alpha]$ be the subspace of $\mathbf{H}_{X}[\alpha]$ consisting of functions supported on the complement of $\bigcup_{\underline{\alpha} \geq n} S_{\underline{\alpha}}$. It is a subspace of $\mathbf{H}_{X}[\alpha]$ of finite codimension. Moreover there are some obvious inclusions $\mathbf{H}_{X}^{<m}[\alpha] \rightarrow \mathbf{H}_{X}^{<n}[\alpha]$ for any $m<n$. Put $\mathbf{H}_{X}^{\geq n}[\alpha]=\mathbf{H}_{X}[\alpha] / \mathbf{H}_{X}^{<n}[\alpha]$. This is a finite dimensional space. We put

$$
\widehat{\mathbf{H}}_{X}[\alpha]=\underset{\operatorname{Lim}}{\longrightarrow} \mathbf{H}_{\bar{X}}^{\geq n}[\alpha], \quad \widehat{\mathbf{H}}_{X}=\bigoplus_{\alpha} \widehat{\mathbf{H}}_{X}[\alpha] .
$$

Note that $\widehat{\mathbf{H}}_{X}[\alpha]=\left\{f: \mathcal{I}_{\alpha} \rightarrow \mathbb{C}\right\}=\prod_{\mathcal{F} \in \mathcal{I}_{\alpha}} \mathbb{C} 1_{\mathcal{F}}$ as a vector space, where we have denoted by $\mathcal{I}_{\alpha} \subset \mathcal{I}_{X}$ the set of all coherent sheaves of class $\alpha$. It is shown in [4, Section 2] that the product and coproduct are well-defined in the limit and endow $\widehat{\mathbf{H}}_{X}$ with the structure of a (twisted) bialgebra.

Consider the elements

$$
\mathbf{1}_{\alpha}=\sum_{\mathcal{F} \in \mathcal{I}_{\alpha}} 1_{\mathcal{F}}, \quad \mathbf{1}_{\alpha}^{\text {vec }}=\sum_{\mathcal{V} \in \mathcal{I}_{\alpha}^{\text {vec }}} 1_{\mathcal{V}}
$$

where the second sum ranges over all (isomorphism classes of) vector bundles of class $\alpha$. These are both elements of $\widehat{\mathbf{H}}_{X}$. As a direct corollary of Proposition 2.3 we have the following identities:

$$
\mathbf{1}_{\alpha}=\sum_{\underline{\alpha} \in X_{\alpha}} v^{\sum_{i<j}\left\langle\alpha_{i}, \alpha_{j}\right\rangle} \mathbf{1}_{\alpha_{1}}^{\mathrm{ss}} \cdots \mathbf{1}_{\alpha_{l}}^{\mathrm{ss}}, \quad \mathbf{1}_{\alpha}^{\mathrm{vec}}=\sum_{\underline{\alpha} \in Y_{\alpha}} v^{\sum_{i<j}\left\langle\alpha_{i}, \alpha_{j}\right\rangle} \mathbf{1}_{\alpha_{1}}^{\mathrm{ss}} \cdots \mathbf{1}_{\alpha_{l}}^{\mathrm{ss}},
$$

where $X_{\alpha}$ is the set of all HN types of weight $\alpha$ and $Y_{\alpha}$ is the set of all HN types $\underline{\alpha}=\left(\alpha_{1}, \ldots, \alpha_{l}\right)$ of weight $\alpha$ for which $\mu\left(\alpha_{l}\right)<\infty$.

\section{Characteristic functions of semistables}

3.1. Our aim in this section is to prove the following theorem:

Theorem 3.1. For any $\alpha \in\left(\mathbb{Z}^{2}\right)^{+}$we have $\mathbf{1}_{\alpha}^{s s} \in \mathbf{U}_{X}$.

Our proof of Theorem 3.1 hinges on the following preliminary result. Let us denote by $\widehat{\mathbf{U}}_{X}$ the completion of $\mathbf{U}_{X}$ in $\widehat{\mathbf{H}}_{X}$ (i.e., $\widehat{\mathbf{U}}_{X}[\alpha]=\operatorname{Lim} \mathbf{U}_{X}[\alpha] /\left(\mathbf{U}_{X}[\alpha]\right.$ $\left.\left.\cap \mathbf{H}_{X}^{<n}[\alpha]\right)\right)$.

Proposition 3.2. For any $\alpha \in\left(\mathbb{Z}^{2}\right)^{+}$we have $\mathbf{1}_{\alpha}^{s s} \in \widehat{\mathbf{U}}_{X}$. 
Proof. We may use Reineke's inversion formula (see [12, Section 5]) to write

$$
\mathbf{1}_{\alpha}^{\mathbf{s s}}=\sum_{\underline{\beta}}(-1)^{s-1} v^{\sum_{i<j}\left\langle\beta_{i}, \beta_{j}\right\rangle} \mathbf{1}_{\beta_{1}} \cdots \mathbf{1}_{\beta_{s}},
$$

where the sum ranges over all tuples $\beta=\left(\beta_{1}, \ldots, \beta_{s}\right)$ of elements of $\left(\mathbb{Z}^{2}\right)^{+}$satisfying $\mu\left(\sum_{l=k}^{s} \beta_{l}\right)>\mu(\alpha)$ for all $k=\overline{1}, \ldots, s$. The above sum converges in $\widehat{\mathbf{H}}_{X}$. Since $\widehat{\mathbf{U}}_{X}$ is a subalgebra of $\widehat{\mathbf{H}}_{X}$, the proposition will be proved if we can show that $\mathbf{1}_{\alpha} \in \widehat{\mathbf{U}}_{X}$ for all $\alpha$. Furthermore, because $\mathbf{1}_{\alpha}=\sum_{l \geq 0} v^{l \operatorname{rank}(\alpha)} \mathbf{1}_{\alpha-(0, l)}^{\mathrm{vec}} \mathbf{1}_{(0, l)}$ and $\mathbf{1}_{(0, l)} \in \mathbf{U}_{X}$ for all $l$, it suffices in fact to prove that $\mathbf{1}_{\alpha}^{\mathbf{v e c}} \in \widehat{\mathbf{U}}_{X}$ for all $\alpha$.

Let us write $\alpha=(r, d)$ and argue by induction on the rank $r$. The cases $r=0,1$ are obvious so let $r>1$ and let us assume that

$$
\mathbf{1}_{\left(r^{\prime}, d\right)} \in \widehat{\mathbf{U}}_{X}, \quad \mathbf{1}_{\left(r^{\prime}, d\right)}^{\text {vec }} \in \widehat{\mathbf{U}}_{X}
$$

for all $r^{\prime}<r$. We have to show that for any $d \in \mathbb{Z}$ and any $n \in \mathbb{Z}$ it holds

$$
\mathbf{1}_{r, d}^{\text {vec }} \in \mathbf{U}_{X}+\mathbf{H}_{X}^{<n} .
$$

Let us fix $n$ and argue by induction on $d$. If $d<n r$, then no vector bundle of rank $r$ and degree $d$ may belong to $\mathbf{C}_{\geq n}$ and hence have an HN type $\underline{\alpha} \geq n$. Therefore $\mathbf{1}_{r, d}^{\text {vec }} \in \mathbf{H}_{X}^{<n}$. Now let us fix some $d$ and assume that (3.2) holds for all $d^{\prime}<d$.

Choose $N<n-2(g-1)$ and let us consider the product $\mathbf{1}_{r-1, d-N} \cdot \mathbf{1}_{1, N}^{\text {vec }}$. By definition, we have

$$
\mathbf{1}_{r-1, d-N} \cdot \mathbf{1}_{1, N}^{\text {vec }}=\sum_{\mathcal{F}} c_{\mathcal{F}}[\mathcal{F}]
$$

where

$$
\begin{aligned}
c_{\mathcal{F}} & =v^{-\langle(r-1, d-N),(1, N)\rangle} \sum_{\mathcal{L} \in \operatorname{Pic}^{N}(X)} \frac{\#\{\mathcal{L} \hookrightarrow \mathcal{F}\}}{\# \operatorname{Aut}(\mathcal{L})} \\
& =v^{-\langle(r-1, d-N),(1, N)\rangle} \sum_{\mathcal{L} \in P i c^{N}(X)} \frac{\#\{\mathcal{L} \hookrightarrow \mathcal{F}\}}{v^{-2}-1} .
\end{aligned}
$$

Let us decompose $\mathcal{F}=\mathcal{V}_{\mathcal{F}} \oplus \mathcal{T}_{\mathcal{F}}$ into a direct sum of a vector bundle and a torsion sheaf, and let us assume that $\mathcal{F} \in \mathbf{C}_{\geq n}$. Then $\mathcal{F} \in \mathbf{C}_{\geq 2(g-1)+N}$ and thus $\operatorname{Ext}(\mathcal{L}, \mathcal{F})=0$ by Serre duality. This in turn implies that $\operatorname{dim} \operatorname{Hom}(\mathcal{L}, \mathcal{F})=$ $\langle(1, N),(r, d)\rangle$. Any nonzero map from a line bundle to a vector bundle is an embedding. From this we deduce that

$\#\{\mathcal{L} \hookrightarrow \mathcal{F}\}=v^{-2 \operatorname{dim} \operatorname{Hom}(\mathcal{L}, \mathcal{F})}-v^{-2 \operatorname{dim} \operatorname{Hom}\left(\mathcal{L}, \mathcal{T}_{\mathcal{F}}\right)}=v^{-2\langle(1, N),(r, d)\rangle}-v^{-2 \operatorname{deg}\left(\mathcal{T}_{\mathcal{F}}\right)}$.

The important point is that this only depends on $\operatorname{deg}\left(\mathcal{T}_{\mathcal{F}}\right)$. From this discussion we deduce that there exists nonzero constants $c_{l}$ for $l \geq 0$ such that

$$
\mathbf{1}_{r-1, d-N} \cdot \mathbf{1}_{1, N}^{\mathbf{v e c}} \in c_{0} \mathbf{1}_{r, d}^{\text {vec }}+\sum_{l=1}^{d-r n} c_{l} \mathbf{1}_{r, d-l}^{\text {vec }} \cdot \mathbf{1}_{0, l}+\mathbf{H}_{X}^{<n} .
$$


We may rewrite this last equation as

$$
c_{0} \mathbf{1}_{r, d}^{\mathbf{v e c}} \in \mathbf{1}_{r-1, d-N} \cdot \mathbf{1}_{1, N}^{\mathbf{v e c}}-\sum_{l=1}^{d-r n} c_{l} \mathbf{1}_{r, d-l}^{\mathbf{v e c}} \cdot \mathbf{1}_{0, l}+\mathbf{H}_{X}^{<n} .
$$

Now, by our two induction hypotheses we have $\mathbf{1}_{r-1, d-N} \in \widehat{\mathbf{U}}_{X}$ and $\mathbf{1}_{r, d-l}^{\text {vec }} \in$ $\widehat{\mathbf{U}}_{X}$ for all $l \geq 1$. But then (3.2) holds as well. We are done.

Proof of Theorem 3.1. We have to show that $\mathbf{1}_{\alpha}^{\mathbf{s s}}$ belongs to $\mathbf{U}_{X}$, and not only to $\widehat{\mathbf{U}}_{X}$. By Proposition 3.2, there exists for all $n$ an element $v_{n} \in \mathbf{H}_{X}^{<n}$ such that $u_{n}:=\mathbf{1}_{\alpha}^{\text {ss }}+v_{n} \in \mathbf{U}_{X}$. We may further decompose $v_{n}=\sum_{\alpha} v_{n, \underline{\alpha}}$ according to the HN type $\underline{\alpha}$. The set of $\underline{\alpha}$ for which $v_{n, \underline{\alpha}}$ is nonzero is finite since $v_{n} \in \mathbf{H}_{X}$. Our proof is based on the following two lemmas.

Lemma 3.3. There exists $n \ll 0$ such that for any $H N$ type $\underline{\alpha}=\left(\alpha_{1}, \ldots, \alpha_{l}\right)$ of weight $\alpha$ satisfying $\mu\left(a_{1}\right)<n$, we have $\mu\left(\alpha_{i+1}\right)-\mu\left(\alpha_{i}\right)>2(g-1)$ for some $1 \leq i \leq l$.

Proof. Let $\underline{\alpha}=\left(\alpha_{1}, \ldots, \alpha_{l}\right)$ be as above. We have $\operatorname{deg}(\alpha)=\operatorname{rank}\left(\alpha_{1}\right) \mu\left(\alpha_{1}\right)+$ $\cdots+\operatorname{rank}\left(\alpha_{l}\right) \mu\left(\alpha_{l}\right)$. If $\mu\left(\alpha_{1}\right)<n$ and $\mu\left(\alpha_{i+1}\right)-\mu\left(\alpha_{i}\right) \leq 2(g-1)$ for all $i$, then

$$
\begin{aligned}
\operatorname{deg}(\alpha)< & \operatorname{rank}\left(\alpha_{1}\right) n+r k\left(\alpha_{2}\right)(n+2(g-1)) \\
& +\cdots+\operatorname{rank}\left(\alpha_{l}\right)(n+2(g-1)(l-1) \\
= & \operatorname{rank}(\alpha) n+\sum_{i=2}^{l} 2(g-1)(l-1) \operatorname{rank}\left(\alpha_{i}\right) \\
< & \operatorname{rank}(\alpha)\left(n+2(g-1) \sum_{l=1}^{\operatorname{rank}(\alpha)} l\right) .
\end{aligned}
$$

This is impossible for $n$ sufficiently negative.

Lemma 3.4. Let $\mathcal{F} \in \operatorname{Coh}(X)$ be a coherent sheaf of class $\alpha$ and of $H N$ type $\left(\alpha_{1}, \ldots, \alpha_{l}\right)$. Assume that $\mu\left(\alpha_{i+1}\right)-\mu\left(\alpha_{i}\right)>2(g-1)$ for some $i$. Then $1_{\mathcal{F}}=m \circ \Delta_{\beta, \gamma}\left(1_{\mathcal{F}}\right)$ for $\beta=\alpha_{1}+\cdots+\alpha_{i}, \gamma=\alpha_{i+1}+\cdots+\alpha_{l}$.

Proof. Let $\mathcal{F}_{l} \subset \cdots \subset \mathcal{F}_{1}=\mathcal{F}$ be the $\mathrm{HN}$ filtration of $\mathcal{F}$. Since $\mathcal{F}_{i+1} \in$ $\mathbf{C}_{\geq \mu\left(\alpha_{i+1}\right)}$ and $\mathcal{F} / \mathcal{F}_{i+1} \in \mathbf{C}_{\leq \mu\left(a_{i}\right)}$ while $\mu\left(\alpha_{i+1}\right)-\mu\left(\alpha_{i}\right)>2(g-1)$ we have $\operatorname{Ext}\left(\mathcal{F}_{i+1}, \mathcal{F} / \mathcal{F}_{i+1}\right)=0$ (see Proposition 2.1). It follows that $\mathcal{F} \simeq \mathcal{F}_{i+1} \oplus$ $\mathcal{F} / \mathcal{F}_{i+1}$. Moreover, $1_{\mathcal{F} / \mathcal{F}_{i+1}} 1_{\mathcal{F}_{i+1}}=v^{-\left\langle\mathcal{F} / \mathcal{F}_{i+1}, \mathcal{F}_{i+1}\right\rangle_{1}} 1_{\mathcal{F}}$ since there is a unique subsheaf of $\mathcal{F}$ isomorphic to $\mathcal{F}_{i}$. Hence Lemma 3.4 will be proved once we show that $\Delta_{\beta, \gamma}\left(1_{\mathcal{F}}\right)=v^{\left\langle\mathcal{F} / \mathcal{F}_{i+1}, \mathcal{F}_{i+1}\right\rangle} 1_{\mathcal{F} / \mathcal{F}_{i+1}} \otimes 1_{\mathcal{F}_{i+1}}$. But this last equation is a consequence of the fact that there exists a unique subsheaf of $\mathcal{F}$ of class $\gamma$, namely $\mathcal{F}_{i+1}$ (see Proposition 2.2).

We are now ready to finish the proof of Theorem 3.1. Let us choose some $n \ll 0$ as in Lemma 3.3. Let $\mathcal{A}$ be the (finite) set of all $\underline{\alpha}$ for which $v_{n, \underline{\alpha}}$ is nonzero and let $\underline{\alpha}^{0}$ be the lower boundary of the convex hull of elements of $\mathcal{A}$. 


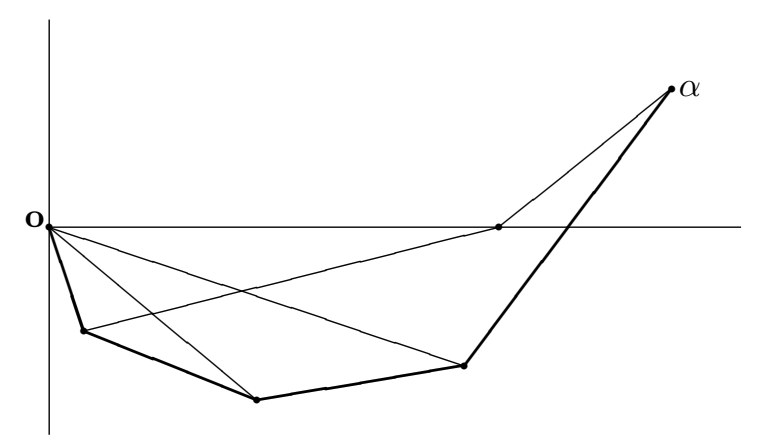

Figure 2. The convex hull of a set of HN polygons.

Thus $\underline{\alpha}^{0}=\left(\alpha_{1}^{0}, \ldots, \alpha_{m}^{0}\right)$ is also a convex path in $\mathbb{Z}^{2}$ of weight $\alpha$. Moreover $\mu\left(\alpha_{1}^{0}\right)<n$ so that the conclusion of Lemma 3.3 applies. Choose $i$ such that $\mu\left(\alpha_{i+1}^{0}\right)-\mu\left(\alpha_{i}^{0}\right)>2(g-1)$ and set $\beta=\alpha_{1}^{0}+\cdots+\alpha_{i}^{0}, \gamma=\alpha_{i+1}^{0}+\cdots+\alpha_{m}^{0}$. By Lemma 3.4, $\Delta_{\beta, \gamma}\left(1_{\mathcal{F}}\right)=0$ for all sheaves $\mathcal{F}$ whose HN polygon doesn't lie below the segment $\beta$.

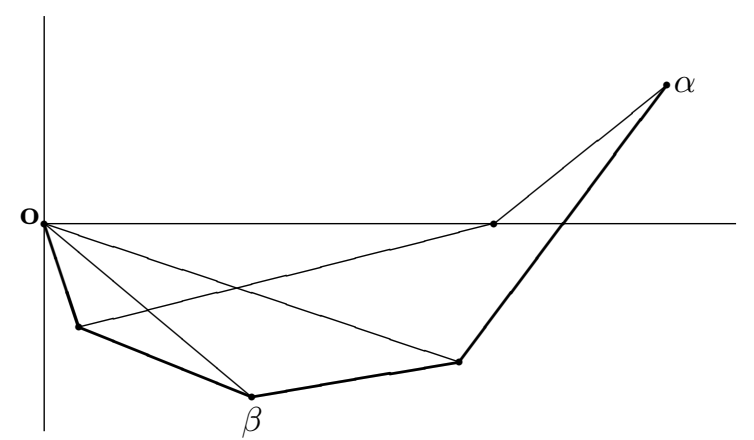

Figure 3. Choice of the vertex $\beta$.

This implies that $\Delta_{\beta, \gamma}\left(v_{n, \underline{\alpha}}\right)=0$ for all HN types $\underline{\alpha}$ whose associated polygon doesn't pass through the point $\beta$. Furthermore, by Lemma 3.4 again, $m \circ \Delta_{\beta, \gamma}\left(v_{n, \underline{\alpha}}\right)=v_{n, \underline{\alpha}}$ for any HN type $\underline{\alpha}$ whose polygon does pass through $\beta$. Hence

$$
m \circ \Delta_{\beta, \gamma}\left(u_{n}\right)=m \circ \Delta_{\beta, \gamma}\left(\mathbf{1}_{\alpha}^{\mathrm{ss}}+\sum_{\underline{\alpha}} v_{n, \underline{\alpha}}\right)=\sum_{\underline{\alpha} \in Z_{\beta}} v_{n, \underline{\alpha}},
$$

where $Z_{\beta}$ is the set of all HN types passing through $\beta$. Because $u_{n}$ belongs to $\mathbf{U}_{X}$, which is stable under the coproduct, we deduce that $\sum_{\underline{\alpha} \in Z_{\beta}} v_{n, \underline{\alpha}}$ belongs to $\mathbf{U}_{X}$ as well. Hence the same holds for $u_{n}^{\prime}=1_{\alpha}^{\text {ss }}+\sum_{\underline{\alpha} \notin Z_{\beta}} v_{n, \underline{\alpha}}$. Notice that $u_{n}^{\prime}$ contains strictly fewer terms than $u_{n}$. Arguing as above repeatedly we obtain better and better approximations of $\mathbf{1}_{\alpha}^{\text {ss }}$ by elements of $\mathbf{U}_{X}$ until we arrive at $\mathbf{1}_{\alpha}^{\text {ss }} \in \mathbf{U}_{X}$. Theorem 3.1 is proved. 
The combination of Theorem 3.1 and Proposition 2.3 yields the following result:

Corollary 3.5. For any $H N$ type $\underline{\alpha}$ we have $\mathbf{1}_{S_{\underline{\alpha}}} \in \mathbf{U}_{X}$.

Remark 3.6. The above proof actually shows that $\widehat{\mathbf{U}}_{X} \cap \mathbf{H}_{X}=\mathbf{U}_{X}$.

\section{Spherical Eisenstein sheaves}

4.1. Let us set $\bar{X}=X \otimes \overline{\mathbb{F}_{q}}$. For $\alpha \in K_{0}^{\prime}(\bar{X})=\mathbb{Z}^{2}$, let $\underline{C o h}{ }_{X}^{\alpha}$ stand for the moduli stack parametrizing coherent sheaves of class $\alpha$ over $\bar{X}$. This is a smooth irreducible stack, which is locally of finite type (see e.g. [9]). It carries a Harder-Narasimhan stratification $\underline{C o h}_{X}^{\alpha}=\bigsqcup_{\alpha} \underline{S}_{\alpha}$ similar to the one existing for $\mathcal{I}_{X}$, and each locally closed substack $\underline{S}_{\alpha}$ is smooth and of finite type.

We will now define, following [16], a certain category of simple perverse sheaves over the stacks $\underline{C o h}_{X}^{\alpha}$. For this we consider the following induction diagrams, for $\alpha, \beta \in K_{0}^{\prime}(X)$ :

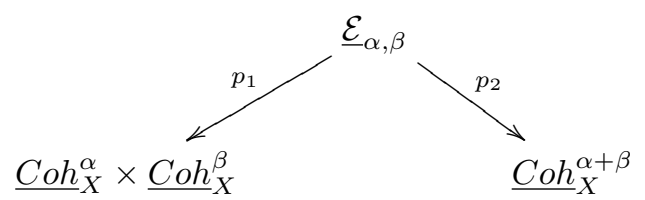

where $\underline{\mathcal{E}}_{\alpha, \beta}$ is the stack classifying inclusions $\mathcal{G} \subset \mathcal{F}$ of a coherent sheaf $\mathcal{G}$ over $\bar{X}$ of class $\beta$ into a coherent sheaf $\mathcal{F}$ over $\bar{X}$ of class $\alpha+\beta$; and where the maps $p_{1}, p_{2}$ are given by the functors $\mathcal{G} \subset \mathcal{F} \mapsto(\mathcal{F} / \mathcal{G}, \mathcal{G})$ and $\mathcal{G} \subset \mathcal{F} \mapsto \mathcal{F}$. The morphism $p_{1}$ is smooth while $p_{2}$ is proper and representable (see [9]).

Let $D^{b}\left(\underline{C o h}_{X}^{\alpha}\right)$ be the bounded derived category of constructible $\overline{\mathbb{Q}}_{l}$-sheaves over $\underline{C o h}_{X}^{\alpha}$. We define induction and restriction functors as

$$
\begin{aligned}
\underline{m}: D^{b}\left(\underline{C o h}_{X}^{\alpha} \times \underline{C o h}_{X}^{\beta}\right) & \rightarrow D^{b}\left(\underline{C o h}_{X}^{\alpha+\beta}\right) \\
\mathbb{P} & \mapsto p_{2} ! p_{1}^{*}(\mathbb{P})\left[\operatorname{dim} p_{1}\right],
\end{aligned}
$$

and

$$
\begin{aligned}
\Delta: D^{b}\left(\underline{\operatorname{Coh}}_{X}^{\alpha+\beta}\right) & \rightarrow D^{b}\left(\underline{C o h}_{X}^{\alpha} \times \underline{C o h}_{X}^{\beta}\right) \\
\mathbb{P} & \mapsto p_{1 !} p_{2}^{*}(\mathbb{P})\left[\operatorname{dim} p_{2}\right] .
\end{aligned}
$$

By the Decomposition Theorem of [3], $\underline{m}$ preserves the subcategory of semisimple complexes of geometric origin. Both of the above functors are associative in the appropriate sense. We will sometimes write $\mathbb{P} \star \mathbb{Q}$ for $\underline{m}(\mathbb{P} \otimes \mathbb{Q})$. For $\alpha \in K_{0}^{\prime}(X)$, let $\mathbf{1}_{\alpha}=\overline{\mathbb{Q}}_{l \underline{C o h}_{X}^{\alpha}}\left[\operatorname{dim} \underline{C o h}_{X}^{\alpha}\right]$ be the constant complex over $\underline{C o h}_{X}^{\alpha}$. We will call a product of the form

$$
L_{\alpha_{1}, \ldots, \alpha_{r}}=\mathbf{1}_{\alpha_{1}} \star \cdots \star \mathbf{1}_{\alpha_{r}}
$$

a Lusztig sheaf. It is a semisimple complex. We let $\mathcal{P}_{X}=\bigsqcup_{\alpha} \mathcal{P}^{\alpha}$ stand for the set of all simple perverse sheaves appearing in some Lusztig sheaf $L_{\alpha_{1}, \ldots, \alpha_{r}}$ 
where for all $\alpha_{i}=\left(r_{i}, d_{i}\right)$ we have $r_{i} \leq 1$. We denote by $\mathcal{Q}_{X}=\bigsqcup_{\alpha} \mathcal{Q}^{\alpha}$ the additive category generated by the objects of $\mathcal{P}_{X}$ and their shifts.

\section{IC sheaves of Harder-Narasimhan strata}

The purpose of this section is to prove the following result:

Theorem 5.1. For any Harder-Narasimhan type $\underline{\alpha}$ we have $I C\left(\underline{S}_{\underline{\alpha}}\right) \in \mathcal{P}_{X}$.

This can be viewed as a direct geometric analog of Theorem 3.1. We will first establish the following special case:

Proposition 5.2. For any $\alpha \in K_{0}^{\prime}(X)$ we have $\mathbf{1}_{\alpha} \in \mathcal{P}_{X}$.

Proof. We argue by induction on the rank $r$ of $\alpha$. If $r=1$, then $\alpha=(1, d)$ for some $d$ and by definition we have $\mathbf{1}_{\underline{C o h}_{X}^{(1, d)}} \in \mathcal{P}_{X}$. Let us fix some $\alpha$ of rank $r>1$ and let us assume that $\mathbf{1}_{\beta}$ belongs to $\mathcal{P}_{X}$ for all $\beta$ of rank strictly less than $r$. Let us choose some $d \ll \mu(\alpha)$ and set $\beta=\alpha-(1, d)$. Consider the convolution diagram

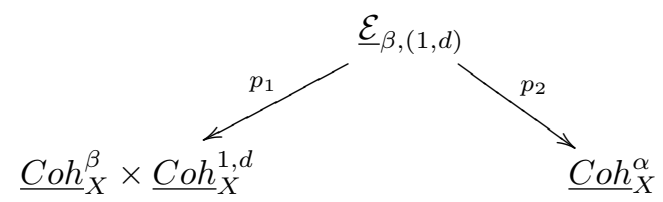

and the corresponding product $\mathbf{1}_{\beta} \star \mathbf{1}_{(1, d)}=p_{2 !}\left(\mathbf{1}_{\left.\underline{\mathcal{E}}_{\beta,(1, d)}\right)}\right)$. By the induction hypothesis, both $\mathbf{1}_{\beta}$ and $\mathbf{1}_{(1, d)}$ belong to $\mathcal{P}_{X}$. Hence it is enough to show that $\operatorname{Hom}\left(\mathbf{1}_{\alpha}, \mathbf{1}_{\beta} \star \mathbf{1}_{(1, d)}\right) \neq\{0\}$. The strata $\underline{S}_{\alpha} \subset \underline{C o h_{X}^{\alpha}}$ is open and we have $\mathbf{1}_{\alpha}=I C\left(\underline{S}_{\alpha}\right)$. Because the complex $\mathbf{1}_{\beta} \star \mathbf{1}_{(1, d)}$ is semisimple, it is sufficient to prove that

$$
\operatorname{Hom}\left(\mathbf{1}_{\underline{S}_{\alpha}},\left(\mathbf{1}_{\beta} \star \mathbf{1}_{(1, d)}\right)_{\underline{S}_{\alpha}}\right)=\operatorname{Hom}\left(\mathbf{1}_{\underline{S}_{\alpha}}, p_{2 !}\left(\mathbf{1}_{\left.\underline{\mathcal{E}}_{\beta,(1, d)}\right)}\right)_{\underline{S}_{\alpha}}\right) \neq\{0\} .
$$

Consider the cartesian diagram obtained by restricting (5.1) to the open strata $\underline{S}_{\alpha}$ :

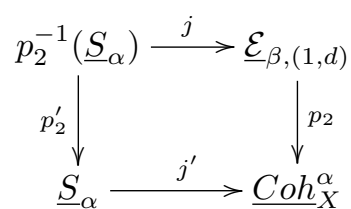

Here $j, j^{\prime}$ are the open embeddings. We now describe the map $p_{2}^{\prime}: p_{2}^{-1}\left(\underline{S}_{\alpha}\right) \rightarrow$ $\underline{S}_{\alpha}$ explicitly. Observe that any semistable sheaf $\mathcal{F}$ of class $\alpha$ is a vector bundle, and hence any subsheaf $\mathcal{G} \subset \mathcal{F}$ of class $(1, d)$ is a line bundle. Let $\underline{\mathcal{H}}$ be the Hom-stack over $\underline{\operatorname{Pic}}{ }^{d} \bar{X} \times \underline{S}_{\alpha}$, i.e., the stack parametrizing triples $(\mathcal{F}, \mathcal{G}, f)$ where $\mathcal{F} \in S_{\alpha} \bar{X}, \mathcal{G} \in \operatorname{Pic}{ }^{d} \bar{X}$ and $f \in \operatorname{Hom}(\mathcal{G}, \mathcal{F})$. Let $a: \underline{\mathcal{H}}^{\prime} \underline{\operatorname{Pic}}^{d} \bar{X} \times \underline{S}_{\alpha}$ be the projection. Observe that since $d \ll \alpha$ and since $\overline{\mathcal{F}}$ is semistable, we have $\operatorname{dim} \operatorname{Hom}(\mathcal{G}, \mathcal{F})=\operatorname{dim} \operatorname{Hom}(\mathcal{G}, \mathcal{F})-\operatorname{dim} \operatorname{Ext}(\mathcal{G}, \mathcal{F})=\langle(1, d), \alpha\rangle$ for any $\mathcal{F}, \mathcal{G}$ 
as above. It follows that $a$ is a vector bundle of $\operatorname{rank}\langle(1, d), \alpha\rangle$. Denote by $a^{\prime}: \underline{\mathcal{H}}^{\prime} \rightarrow \underline{P i c}{ }^{d} \bar{X} \times \underline{S}_{\alpha}$ the associated projective bundle. It is easy to see that $p_{2}^{\prime}: \bar{p}_{2}^{-1}\left(\underline{S}_{\alpha}\right) \rightarrow \underline{S}_{\alpha}$ is canonically isomorphic to the composition of $a^{\prime}$ with the projection $\pi: \underline{\operatorname{Pic}}^{d} \bar{X} \times \underline{S}_{\alpha} \rightarrow \underline{S}_{\alpha}$.

We may now compute

$$
\left(\mathbf{1}_{\beta} \star \mathbf{1}_{(1, d)}\right)_{\mid \underline{S}_{\alpha}}=\left(j^{\prime}\right)^{*} p_{2 !}\left(\mathbf{1}_{\underline{\mathcal{E}}_{\beta,(1, d)}}\right)=p_{2 !}^{\prime}\left(\mathbf{1}_{p_{2}^{-1}\left(\underline{S}_{\alpha}\right)}\right)=\pi_{!} a_{!}^{\prime}\left(\mathbf{1}_{\underline{\mathcal{H}}^{\prime}}\right) .
$$

Because $a^{\prime}$ is a projective bundle, the constant sheaf $\mathbf{1}_{\underline{P i c}} \bar{X} \times \underline{S}_{\alpha}$ appears as a direct summand of $a_{!}^{\prime}\left(\mathbf{1}_{\underline{\mathcal{H}}^{\prime}}\right)$. Therefore $\mathbf{1}_{\underline{S}_{\alpha}}$ appears as a direct summand of $\pi_{!} a_{!}^{\prime}\left(\mathbf{1}_{\mathcal{H}^{\prime}}\right)$. This shows $(5.2)$ and finishes the proof of Proposition 5.2.

We are now in position to prove Theorem 5.1. Let $\underline{\alpha}=\left(\alpha_{1}, \ldots, \alpha_{l}\right)$ be some HN type of weight $\alpha=\sum \alpha_{i}$. By Proposition 5.2, all the perverse sheaves $I C\left(\underline{S}_{\alpha_{i}}\right)=\mathbf{1}_{\alpha_{i}}$ belong to $\mathcal{P}_{X}$. We will show that $I C\left(\underline{S}_{\alpha}\right)$ belongs to $\mathcal{P}_{X}$ as well by proving that

$$
\operatorname{Hom}\left(I C\left(\underline{S}_{\underline{\alpha}}\right), \mathbf{1}_{\alpha_{1}} \star \cdots \star \mathbf{1}_{\alpha_{l}}\right) \neq\{0\} .
$$

For this, consider the (iterated) induction diagram:

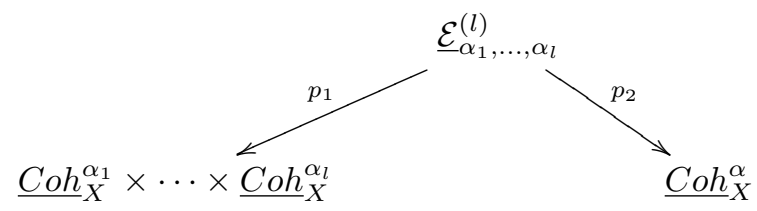

We claim that $\underline{S}_{\underline{\alpha}}$ is open and dense in $\operatorname{Im}\left(p_{2}\right)$. Indeed, set $\underline{\mathcal{E}}_{\alpha_{1}, \ldots, \alpha_{l}}^{(l), 0}=$ $p_{1}^{-1}\left(\underline{S}_{\alpha_{1}} \times \cdots \underline{S}_{\alpha_{l}}\right)$. It is an open dense subset of $\underline{\mathcal{E}}_{\alpha_{1}, \ldots, \alpha_{l}}^{(l)}$ and by construction $p_{2}\left(\underline{\mathcal{E}}_{\alpha_{1}, \ldots, \alpha_{l}}^{(l), 0}\right)=\underline{S}_{\underline{\alpha}}$. Since $p_{2}$ is continuous, $p_{2}^{-1}\left(\underline{\operatorname{Coh}}_{X}^{\alpha} \backslash \underline{\underline{S}_{\alpha}}\right)$ is an open substack of $\underline{\mathcal{E}}_{\alpha_{1}, \ldots, \alpha_{l}}^{(l)}$ which does not intersect $\underline{\mathcal{E}}_{\alpha_{1}, \ldots, \alpha_{l}}^{(l), 0}$. But this means that $p_{2}^{-1}\left(\underline{C o h}_{X}^{\alpha} \backslash \underline{S}_{\underline{\alpha}}\right)$ is empty, i.e., that $\operatorname{Im}\left(p_{2}\right) \subset \underline{S}_{\underline{\alpha}}$ as wanted.

By definition, $\mathbf{1}_{\alpha_{1}} \star \cdots \star \mathbf{1}_{\alpha_{l}}=p_{2 !}\left(\mathbf{1}_{\underline{\mathcal{E}}_{\alpha_{1}, \ldots, \alpha_{l}}^{(l)}}\right)$. This is a semisimple complex and, by the above, $\underline{S}_{\underline{\alpha}}$ is open in its support. Therefore

$$
\begin{aligned}
\operatorname{Hom}\left(I C\left(\underline{S}_{\underline{\alpha}}\right), \mathbf{1}_{\alpha_{1}} \star \cdots \star \mathbf{1}_{\alpha_{l}}\right) & =\operatorname{Hom}\left(\mathbf{1}_{\underline{S}}, j_{\underline{\alpha}}^{*}\left(\mathbf{1}_{\alpha_{1}} \star \cdots \mathbf{1}_{\alpha_{l}}\right)\right) \\
& =\operatorname{Hom}\left(\mathbf{1}_{\underline{S}}, j_{\underline{\alpha}}^{*} p_{2 !}\left(\mathbf{1}_{\underline{\mathcal{E}}_{\alpha_{1}, \ldots, \alpha_{l}}^{(l)}}\right)\right),
\end{aligned}
$$

where $j_{\underline{\alpha}}: \underline{S}_{\alpha} \rightarrow \underline{C o h}_{X}^{\alpha}$ denote the inclusion. Observe that by the uniqueness of the Harder-Narasimhan filtration $\mathcal{F}_{l} \subset \cdots \subset \mathcal{F}_{1}=\mathcal{F}$ of a coherent sheaf $\mathcal{F} \in S_{\underline{\alpha}}$, the projective map $p_{2}$ restricts to an isomorphism $p_{2}^{-1}\left(\underline{S}_{\alpha}\right) \stackrel{\sim}{\rightarrow} \underline{S}_{\underline{\alpha}}$. By base change, $j_{\underline{\alpha}}^{*} p_{2 !}\left(\mathbf{1}_{\underline{\mathcal{E}}_{\alpha_{1}, \ldots, \alpha_{l}}^{(l)}}\right)=\mathbf{1}_{\underline{S} \underline{\alpha}}$. But then $\operatorname{Hom}\left(I C\left(\underline{S}_{\underline{\alpha}}\right), \mathbf{1}_{\alpha_{1}} \star \cdots \star \mathbf{1}_{\alpha_{l}}\right)=$ $\overline{\mathbb{Q}}_{l}$, and (5.4) follows. Theorem 5.1 is proved. 
Remark 5.3. The collection of simple perverse sheaves $\left\{I C\left(\underline{S}_{\alpha}\right\}_{\alpha}\right.$ by no means exhausts of all $\mathcal{Q}_{X}$. We illustrate this by a very simple example showing that one has at least to consider the various Brill-Noether stratas $\underline{W}_{r, d}^{k}$ of the stacks $\underline{C o h}_{X}^{r, d}$ (see e.g. [1] or [8]; note that we use slightly different notation). Let $(r, d)=(2,0)$, and let us consider the direct summands of $\mathbf{1}_{(1,0)} \star \mathbf{1}_{(1,0)}$. The stack $\underline{S}_{2,0} \subset \underline{C o h}_{X}^{2,0}$ of semistable bundles may be stratified as follows: $\underline{S}_{2,0}=$ $\underline{W}_{2,0}^{0} \cup \underline{W}_{(2,0)}^{1} \cup \underline{W}_{2,0}^{2} \cup \underline{U}$ where

$$
\begin{gathered}
\underline{W}_{2,0}^{0}=\left\{\mathcal{V} \in S_{2,0} \mid \operatorname{Hom}(\mathcal{L}, \mathcal{V})=\{0\}, \forall \mathcal{L} \in \operatorname{Pic} c^{0} \bar{X}\right\}, \\
\underline{W}_{2,0}^{1}=\left\{\mathcal{V} \in S_{2,0} \mid \exists ! \mathcal{L} \in \operatorname{Pic} c^{0} \bar{X}, \operatorname{Hom}(\mathcal{L}, \mathcal{V})=\overline{\mathbb{F}_{q}}\right\}, \\
\underline{U}=\left\{\mathcal{V} \in S_{2,0} \mid \exists \mathcal{L}, \mathcal{L}^{\prime} \in \operatorname{Pic} c^{0} \bar{X}, \mathcal{L} \neq \mathcal{L}^{\prime}, \mathcal{V} \simeq \mathcal{L} \oplus \mathcal{L}^{\prime}\right\}, \\
\underline{W}_{2,0}^{2}=\left\{\mathcal{V} \in S_{2,0} \mid \exists ! \mathcal{L} \in P i c^{0} \bar{X}, \operatorname{Hom}(\mathcal{L}, \mathcal{V})={\overline{\mathbb{F}_{q}}}^{2}\right\} .
\end{gathered}
$$

The strata $\underline{W}_{2,0}^{1}$ consists of semistable vector bundles which are nontrivial extensions

$$
0 \longrightarrow \mathcal{L} \longrightarrow \mathcal{V} \longrightarrow \mathcal{L}^{\prime} \longrightarrow 0
$$

of two degree zero line bundles $\mathcal{L}, \mathcal{L}^{\prime}$; the strata $\underline{W}_{2,0}^{2}$ consists of semistable bundles of the form $\mathcal{V} \simeq \mathcal{L}^{\oplus 2}$ for some degree zero line bundle $\mathcal{L}$. Moreover, $\underline{W}_{2,0}^{0}$ is open dense, $\underline{W}_{2,0}^{2}$ is closed and we have inclusions of strata closures $\underline{W_{2,0}^{1}} \supset \underline{\bar{U}} \supset \underline{W}_{2,0}^{2}$.

The restriction

$$
p_{2}^{\prime}: p_{2}^{-1}\left(\underline{S}_{2,0}\right) \rightarrow \underline{S}_{2,0}
$$

of the proper map $p_{2}$ in the induction diagram (4.1) corresponding to $\mathbf{1}_{(1,0)}$ * $\mathbf{1}_{(1,0)}$ respects the above stratification. The following table lists the dimension of each strata as well as the type of fiber:

\begin{tabular}{|c|c|c|}
\hline Strata & Dimension & Fiber \\
\hline$\underline{W}_{2,0}^{0}$ & $4 g-4$ & $\emptyset$ \\
\hline$\underline{W_{2,0}^{1}}$ & $3 g-3$ & $\{p t\}$ \\
\hline$\underline{U}$ & $2 g-2$ & $\{p t\} \cup\{p t\}$ \\
\hline$\underline{W_{2,0}^{2}}$ & $g-4$ & $\mathbb{P}^{1}$ \\
\hline
\end{tabular}

It follows from the above table that $p_{2}^{\prime}$ is a small resolution of the closure $\overline{W_{2,0}^{1}}$ of $\underline{W}_{2,0}^{1}$, and hence that $p_{2 !}^{\prime}\left(\mathbf{1}_{p_{2}^{-1}\left(\underline{S}_{2,0}\right)}\right)=I C\left(\underline{W}_{2,0}^{1}\right)$. Considering induction products of the form $\mathbf{1}_{1, d} \star \mathbf{1}_{1,-d}$ for $d=1, \ldots, 2 g-2$ we obtain elements of $\mathcal{P}_{X}$ supported on other nontrivial Brill-Noether type stratas of $\underline{S}_{2,0}$. 
Remark 5.4. The method of proof of Theorem 5.1 is readily transposable to the context of quivers. Let $\vec{Q}$ be a quiver and let us assume that it contains no oriented cycles. Lusztig defined in [10] a set $\mathcal{Q}_{\vec{Q}}$ of simple perverse sheaves over the moduli stacks $\underline{\mathcal{M}}_{\vec{Q}}^{\alpha}, \alpha \in K_{0}(\vec{Q})$. To any linear form $\Theta: K_{0}(\vec{Q}) \rightarrow \mathbb{C}$ (the 'stability parameter') one may attach a slope function $\mu_{\Theta}$ on $K_{0}(\vec{Q})$ and a Harder-Narasimhan stratification $\underline{\mathcal{M}}_{\vec{Q}}^{\alpha}=\bigsqcup_{\underline{\alpha}} \underline{S}_{\underline{\alpha}}$ (see [12]). Then

Theorem 5.5. For any stability parameter $\Theta$ and any Harder-Narasimhan strata $\underline{S}_{\underline{\alpha}}$ we have $\operatorname{IC}\left(\underline{S}_{\underline{\alpha}}\right) \in \mathcal{P}_{\vec{Q}}$.

Note that the analog of Proposition 5.2 holds since $\vec{Q}$ has no oriented cycles. Theorem 3.1 also holds in the context of quivers, where it is a simple consequence of Reineke's inversion formula ([12]).

\section{References}

1] E. Arbarello, M. Cornalba, P. Griffiths, and J. Harris, Geometry of algebraic curves. Volume I, Grundlehren Math. Wiss. 267, Springer-Verlag, 1985.

[2] P. Baumann and C. Kassel, The Hall algebra of the category of coherent sheaves on the projective line, J. Reine Angew. Math. 533 (2001), 207-233.

[3] A. Beilinson, I. Bernstein, and P. Deligne, Faisceaux pervers, Analysis and topology on singular spaces, I (Luminy, 1981), 5-171, Astérisque, 100, Soc. Math. France, Paris, 1982.

[4] I. Burban and O. Schiffmann, On the Hall algebra of an elliptic curve, I., preprint math.AG/0505148, to appear in Duke Math. Jour.

[5] G. Harder and M. S. Narasimhan, On the cohomology groups of moduli spaces of vector bundles on curves, Math. Ann. 212 (1974/75), 215-248.

[6] M. Kapranov, Eisenstein series and quantum affine algebras, Algebraic geometry 7, J. Math. Sci. (New York) 84 (1997), no. 5, 1311-1360.

[7] M. Kashiwara and Y. Saito, Geometric construction of crystal bases, Duke Math. J. 89 (1997), no. 1, 9-36.

[8] A. King, A survey of Brill-Noether theory on algebraic curves, preprint (1993), available at http://www.maths.bath.ac.uk/ masadk/papers/.

[9] G. Laumon, Faisceaux automorphes liés aux séries d'Eisenstein, Automorphic forms, Shimura varieties, and L-functions, Vol. I (Ann Arbor, MI, 1988), 227-281, Perspect. Math., 10, Academic Press, Boston, MA, 1990.

[10] G. Lusztig, Introduction to Quantum Groups, Birkhaüser, 1994.

[11] H. Nakajima, Quiver varieties and Kac-Moody algebras, Duke Math. J. 91 (1998), no. $3,515-560$.

[12] M. Reineke, The Harder-Narasimhan system in quantum groups and cohomology of quiver moduli, Invent. Math. 152 (2003), no. 2, 349-368.

[13] C. Ringel, Hall algebras and quantum groups, Invent. Math. 101 (1990), no. 3, 583-591.

[14] O. Schiffmann, Noncommutative projective curves and quantum loop algebras, Duke Math. J. 121 (2004), no. 1, 113-168.

[15] _ Lectures on Hall algebras, preprint arXiv:math/0611617 (2006), to appear.

[16] _ Canonical bases and moduli spaces of sheaves on curves, Invent. Math. 165 (2006), no. 3, 453-524.

[17] On the Hall algebra of an elliptic curve, II, preprint arXiv:math/0508553 $(2005)$. 
[18] O. Schiffmann and E. Vasserot, The elliptic Hall algebra, Cherednick Hecke algebras and Macdonald polynomials, Compos. Math. 147 (2011), no. 1, 188-234.

[19] _ Hall algebras of curves, quiver varieties and Langlands duality, arXiv:1009.0678 (2010).

[20] S. Shatz, The decomposition and specialization of algebraic families of vector bundles, Compositio Math. 35 (1977), no. 2, 163-187.

[21] M. Varagnolo and E. Vasserot, On the decomposition matrices of the quantized Schur algebra, Duke Math. J. 100 (1999), no. 2, 267-297.

Département de Mathématiques

BÂtiment 425 FACUlté des Sciences d'Orsay

Université PARIS-SUd 11

F-91405 Orsay Cedex, France

E-mail address: Olivier.Schiffmann@math.u-psud.fr 